\title{
Neuroprotective effects of curcumin against rats with focal cerebral ischemia-reperfusion injury
}

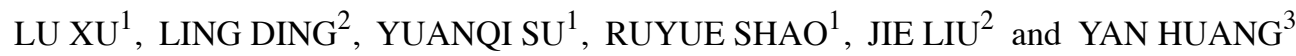 \\ ${ }^{1}$ School of Pharmacy, Chongqing Medical and Pharmaceutical College, Chongqing 401331; ${ }^{2}$ Pharmacy Department, \\ The Central Hospital of Jiangjin, Chongqing 402260; ${ }^{3}$ Scientific Research and Teaching Department, \\ Chongqing Traditional Chinese Medicine Hospital, Chongqing 400021, P.R. China
}

Received October 18, 2018; Accepted February 7, 2019

DOI: $10.3892 /$ ijmm.2019.4094

\begin{abstract}
The aim of the present study was to investigate the protective effects of curcumin and its effect on the methyl ethyl ketone/extracellular signal regulated kinase/cAMP-response element binding protein (MEK/ERK/CREB) pathway. The study was conducted in vivo and in vitro as follows: In vivo: Focal cerebral ischemia-reperfusion (IR) models of rats were made with the plug-line method. Adult male Sprague-Dawley rats were divided into four groups: Sham operation control group, IR and curcumin-treatment groups (100 mg/kg and IC, $300 \mathrm{mg} / \mathrm{kg}$ ). The effects of curcumin on neurological deficit scores, brain water content and infarct volumes were identified. Transmission electron microscope was utilized to observe morphological changes of hippocampal neurons; hematoxylin and eosin staining was used to observe morphological changes of brain tissue; and the terminal deoxynucleotidyl transferase (TdT)-mediated dUTP nick end labeling method detected neurons apoptosis of hippocampal CA1. Finally, western blot analysis detected the expression of phosphorylated (p)-MEK, p-ERK, p-CREB, B-cell lymphoma-2 (Bcl-2) and $\mathrm{Bcl}-2$ associated $\mathrm{X}$ protein (Bax). In vitro: An oxygen-glucose deprivation/reoxygenation method was used on primary cultured astrocytes to make cerebral ischemia-reperfusion models in vitro. Astrocytes were randomly divided into five groups: Normoxia, oxygen-glucose deprivation/reoxygenation (OGD/Reoxy), OGD/Reoxy + curcumin $(5,10,20 \mu \mathrm{mol} / \mathrm{l})$. The cell viability and toxicity were assessed by MTT and
\end{abstract}

Correspondence to: Dr Jie Liu, Pharmacy Department, The Central Hospital of Jiangjin, 725 Jiangzhou Avenue, Dingshan, Chongqing 402260, P.R. China

E-mail: 453039683@qq.com

Dr Yan Huang, Scientific Research and Teaching Department, Chongqing Traditional Chinese Medicine Hospital, No. 6, Panxi 7 Branch Road, Chongqing 400021, P.R. China

E-mail: huangyan1011@163.com

Key words: curcumin, ischemia-reperfusion, astrocyte, methyl ethyl ketone, extracellular signal regulated kinase, cAMP-response element binding protein, B-cell lymphoma-2, B-cell lymphoma-2 associated $\mathrm{X}$ protein lactate dehydrogenase release assay, and levels of p-MEK, p-ERK and p-CREB proteins were analyzed by the western blotting method. Curcumin was demonstrated to improve nerve damage symptoms and infarct volume, reduce brain water content, relieve neuronal apoptosis and also increase the expression of p-MEK, p-ERK, p-CREB, Bcl-2 and reduce Bax levels in vivo and in vitro. In conclusion curcumin can mitigate focal cerebral ischemia-reperfusion injuries and this effect may be carried out through the MEK/ERK/CREB pathway.

\section{Introduction}

Approximately 5 million fatalities globally are attributed to stroke each year. Ischemic stroke accounts for more than $80 \%$ of total stroke cases and is one of the main causes of mortality and disability worldwide (1-4). Following a period of ischemia, severe reperfusion injury occurs when blood returns to the brain. Reperfusion therefore serves a critical role in cerebral ischemia (5). In clinical treatment, thrombolytic therapy is the only method approved for the treatment of ischemic stroke $(6,7)$. However, restoration of blood flow following thrombolytic treatment may cause severe ischemia-reperfusion injury (8). Currently, the neuroprotective approach has become a novel direction in the treatment of stroke and has been studied in animal experiments, but the efficacy on patients remains limited (9). Consequently, there is an urgent need to develop an effective neuroprotective agent for the prevention and treatment of stroke.

In acute ischemic stroke, apoptosis has been considered one of the main causes of neuronal death $(10,11)$. Apoptosis is evident in animal models and in patients of ischemic stroke. Therefore, inhibition of apoptotic pathways and the creation of a neuroprotective environment provide a potential therapeutic approach for ischemic brain damage. Mitogen activated protein kinase (MAPK) is one of the distinct signaling cascades involved in neuroprotection and is activated during the ischemic process (12). MAPK signaling pathway is activated then causes extracellular signal regulated kinase (ERK) phosphorylation induced by methyl ethyl ketone (MEK) (13). It has been demonstrated that activation of MEK/ERK elicits an anti-apoptosis effect during cerebral ischemia (14). cAMP-response element binding protein (CREB) is a typical protein that possesses anti-apoptotic properties (15). 
Down-modulation of the MEK/ERK pathways inhibits the phosphorylation of CREB, which causes B-cell lymphoma-2 (Bcl-2) family transcription and expression downregulation and sensitization to cell apoptosis (16).

Curcumin (Cur) was extracted from the rhizome of Curcuma genus plants (17). Cur has anti-inflammatory, antioxidant, anti-apoptotic and other pharmacological effects (18). In cerebral ischemia-reperfusion injury, Cur is also known to have neuroprotective properties (19). However, the underlying molecular mechanisms are still poorly understood. In this study, the aim was to investigate whether Cur protected the brain from ischemic injury by the MEK/ERK/CREB pathway during in vivo and in vitro experiments. The results of the present study will inform future investigations into the neuroprotective effects of curcumin on the molecular level.

\section{Materials and methods}

Materials and reagents. Cur was purchased from SigmaAldrich; Merck KGaA (Darmstadt, Germany). Antibodies against phosphorylated anti-(p)-MEK), anti-MEK, anti-p-ERK, anti-ERK, anti-CREB, anti-p-CREB, B-cell lymphoma-2 (Bcl-2), Bcl-2 associated $\mathrm{X}$ protein (Bax), $\beta$-actin antibodies and horseradish peroxidase (HRP)-conjugated secondary antibodies were all provided by Sigma-Aldrich (Merck $\mathrm{KGaA}$ ). Terminal dexynucleotidyl transferase (TdT)-mediate $\mathrm{d}$ dUTP nick end labeling (TUNEL) assay kits were obtained from Roche Diagnostics (Basel, Switzerland). Lactate dehydrogenase release assay (LDH) kits were purchased from Nanjing Jiancheng Bioengineering Institute (Nanjing, China). Dulbecco's modified Eagle media: Nutrient Mixture F-12 (DMEM/F12) medium, fetal calf serum and trypsin were purchased from Hyclone (Logan, UT, USA). Unless otherwise stated, triphenyl tetrazolium chloride (TTC) powder, PBS buffer, paraformaldehyde, western blot reagents, tetrazolium blue tetrazolium bromide (MTT) and all other chemicals used were provided by Sigma Aldrich; Merck KGaA.

Animals. A total of 60 male Sprague-Dawley (SD) rats (280-320 g) for in vivo experiment and a total of 5 SD rats (1-2 days old) for primary astrocyte cell culture were provided by the Experimental Animal Center of Chongqing Medical University (Chongqing, China) Specific Pathogen Free [animal production license no. SCXK (Chongqing) 2011-0001]. Animals were kept in a controlled room with adequate food and water, constant temperature and humidity $\left(22^{\circ} \mathrm{C}\right.$ and $55 \%$, respectively) as well as a 12/12 h light/dark cycle.

Establishment of the cerebral ischemia-reperfusion model and animal grouping. Experimental protocols were all approved by the Chongqing Medical University's Institutional Animal Care and Use Committee. Using Longa's method, focal cerebral ischemia and reperfusion of middle cerebral artery occlusion models of rats were performed. Rats were anesthetized with $4 \%$ chloral hydrate through intraperitoneal injection, then an incision in the skin was made to expose the right common carotid artery, internal carotid artery (ICA) and external carotid artery. The right middle cerebral artery occlusion model was established by introducing an embolus through the ICA. The embolus was inserted into the internal carotid artery until the tip reached the origin of the middle cerebral artery $(18-22 \mathrm{~mm})$. After $120 \mathrm{~min}$ of ischemia, the embolus was removed. After the procedure, the rats recovered in pre-warmed cages. In total, 60 rats were divided into 4 groups randomly as follows: i) Sham operation control group (SC) $(n=15)$; ii) ischemia-reperfusion group (IR) ( $\mathrm{n}=15)$; iii) Cur-treatment group (IC) receiving cur at $100 \mathrm{mg} / \mathrm{kg}(\mathrm{n}=15)$; iv) IC receiving Cur at $300 \mathrm{mg} / \mathrm{kg}(\mathrm{n}=15)$. Pre-treatment with Cur took place $30 \mathrm{~min}$ prior to surgery and daily via intraperitoneal injection. The sham group and ischemia-reperfusion group received similar pre-treatment with normal saline.

Evaluation of neurological deficit scores. A total of $24 \mathrm{~h}$ following ischemia/reperfusion, the neurological deficit score was examined by referencing the standard 5 point system established by Longa et al (20): i) No obvious signs of deficit (score 0 ); ii) failure to extend the right forepaw fully (score 1); iii) circling to the right (score 2); iv) falling to the right (score 3); and v) loss of walking abilities (score 4).

Measurement of the brain water content. After evaluation of neurological deficit scores, 3 rats were anesthetized with $4 \%$ chloral hydrate via intraperitoneal injection and rapidly decapitated. Brain tissue was removed quickly, followed by the pia mater and brain tissue blood. Tissue wet weight (A) and dry weight (B, tissue was dried in an oven for $24 \mathrm{~h}$ ) were weighed (accurate to $0.1 \mathrm{mg}$ ). Finally, the brain water content was calculated in accordance with the Blliot formula: Brain water content $=(\mathrm{A}-\mathrm{B}) / \mathrm{A} \times 100 \%$.

Measurement of cerebral infarct volume. Following evaluation of neurological deficit scores, 3 other rats were anesthetized with $4 \%$ chloral hydrate via intraperitoneal injection and rapidly decapitated. The brain was removed and coronally sectioned into $2 \mathrm{~mm}$ slices, which were placed into $2 \%$ TTC solution at $37^{\circ} \mathrm{C}$. After $20 \mathrm{~min}$, slices were stained and images were captured with a camera. Noninfarcted areas of the brain tissue were stained red; infarcted areas appeared white. CMIAS-008 image analysis software (Institute of Beijing University of Aeronautics and Astronautics, Beijing, China) was used to calculate the ratio of the infarct size/the total area.

Transmission electron microscopy (TEM) to observe morphological changes of hippocampal neurons. After evaluation of neurological deficit scores, 3 rats were perfused transcardially with $0.9 \% \mathrm{NaCl}$ and $2.5 \%$ glutaraldehyde and rapidly decapitated. Brain tissue sized $1 \mathrm{~mm}^{3}$ were quickly removed and fixed in $2.5 \%$ glutaraldehyde at $37^{\circ} \mathrm{C}$. After $1 \mathrm{~h}$, specimens were dehydrated with acetone and embedded by Epon 812 at $60^{\circ} \mathrm{C}$ for $24 \mathrm{~h}$. The $60 \mathrm{~nm}$ ultrathin sections of the cubes were stained with uranyl acetate and lead citrate at $25^{\circ} \mathrm{C}$ for $30 \mathrm{~min}$, and then observed under TEM (7100; Hitachi, Tokyo, Japan).

Hematoxylin and eosin staining $(H \& E)$ to observe morphological changes of brain tissue. A total of 3 anesthetized rats were perfused transcardially with $0.9 \% \mathrm{NaCl}$ and $4 \%$ paraformaldehyde through the left ventricle and rapidly decapitated. After brains were removed and embedded in paraffin, they were processed into $5-\mu \mathrm{m}$-thick slices. Finally, H\&E was applied at $25^{\circ} \mathrm{C}$ for $15 \mathrm{~min}$ to the tissue and the pathological changes 
of cells in hippocampal CA1 region were observed under an optical microscope (Olympus Corporation, Tokyo, Japan).

TUNEL to detect neurons apoptosis in hippocampal CA1. TUNEL was used to detect neuron apoptosis in hippocampal CA1 in according to the TUNEL assay kit protocol. Stained slices with $5-\mu \mathrm{m}$-thick were imaged under the optical microscope. The extent of brain damage was then evaluated by apoptotic index, which was the arithmetic mean of positive cells counted in 5 microscopic fields in each CA1 region of the hippocampus section.

Western blot analysis. A total of $24 \mathrm{~h}$ following reperfusion, the hippocampal CA1 of remaining three rats in each group was removed and put on ice. Total protein extraction and protein determination was performed with a protein extraction kit (Beyotime Institute of Biotechnology, Shanghai, China). Subsequently, using western blot analysis, the expression of MEK, p-MEK, p-ERK, ERK, CREB, p-CREB, Bcl-2 and Bax was measured. Protein $(30 \mu \mathrm{l})$ were separated by $12 \%$ SDS-PAGE and transferred onto a nitrocellulose membrane. The membrane was blocked with $10 \%$ goat serum (Beyotime Institute of Biotechnology) at $25^{\circ} \mathrm{C}$ for $2 \mathrm{~h}$ and the anti-rabbit antibodies against MEK (cat. no. SAB4502407), p-MEK (cat. no. HPA026430), ERK (cat. no. M7927), p-ERK (cat. no. M7927), CREB (cat. no. SAB4500444), p-CREB (cat. no. AV01026), Bcl-2 (cat. no. SAB4500003), and anti-mouse antibodies against Bax (cat. no. B8554) and $\beta$-actin (cat. no. A5441) $(1: 1,000)$ were added to the membrane overnight at $4^{\circ} \mathrm{C}$. After washing, the membranes were incubated with anti-rabbit HRP-conjugated secondary antibodies (cat. no. AP182P; 1:2,000) for $40 \mathrm{~min}$ at $37^{\circ} \mathrm{C}$. Subsequently, the membranes were processed with an ECL kit (Beyotime Institute of Biotechnology) to detect immune reactivity. Finally, images were analyzed by a Versa Doc Model 3000 (Bio-Rad Laboratories, Inc., Hercules, CA, USA).

Primary astrocyte cell culture. Cerebral cortexes from 1-2 days old SD rats were separated under sterile conditions. The cell suspensions were seeded $\left(2 \times 10^{6}\right.$ cells $\left./ \mathrm{cm}^{2}\right)$ into culture plates and complete medium was added. Cultured cells were grown in an incubator at $37^{\circ} \mathrm{C}$ with $5 \% \mathrm{CO}_{2}$ and $100 \%$ humidity. After $24 \mathrm{~h}$, astrocytes were replaced with new medium and the medium was replaced every three days. After 11 days astrocytes were used for future experiments. The purity of astrocytes was identified as above $95 \%$ by fluorescent antibody technical analysis with anti-glial fibrillary acidic protein (anti-GFAP).

Establishment of the cerebral ischemia-reperfusion model in vitro and astrocyte grouping. Astrocytes were seeded at a density of $5 \times 10^{4}$ into 24 -well plates and cultured for $24 \mathrm{~h}$ in an incubator $\left(37^{\circ} \mathrm{C}, 5 \% \mathrm{CO}_{2}\right)$. Subsequently, cells were divided into the normoxic group, oxygen-glucose deprivation/reoxygenation group (OGD/Reoxy) and OGD/Reoxy + Cur group (OGD/Reoxy + C group) randomly. There were 6 wells in each group. In the MTT and LDH experiments, the OGD/Reoxy $+\mathrm{C}$ group has been divided into three doses [5, 10, 20 micromolar $(\mu \mathrm{mol}) /$ liter $(1)]$, and setting 6 duplicate holes in each group. The solubilization of Cur was achieved with dimethyl sulfoxide (DMSO), but the final concentration of DMSO did not exceed $0.1 \%$ in the medium. In the drug-administered group, 1,000 $\mu \mathrm{l}$ of complete medium containing the corresponding drug was added, the normal group and the model group cells had 1,000 $\mu$ l of complete medium containing no drug but containing the same amount of solvent (DMSO) added as the administration group. Cells were cultivated for $24 \mathrm{~h}$ in normal medium, then the model group and drug-administered group were modeled: Cells were subjected to hypoxia and hypoglycemia for $2 \mathrm{~h}$ and then reoxygenated for $24 \mathrm{~h}$.

Identification of astrocytes. Cell climbing pieces were washed and fixed by $4 \%$ paraformaldehyde at $25^{\circ} \mathrm{C}$ for $20 \mathrm{~min}$. Then cell climbing pieces were blocked with $5 \%$ goat serum at $37^{\circ} \mathrm{C}$ for $1 \mathrm{~h}$, and the primary antibodies against GFAP (cat. no. G3893) (1:50; Sigma-Aldrich; Merck $\mathrm{KGaA}$ ) were added overnight at $4^{\circ} \mathrm{C}$. After washing, the cell climbing samples were incubated with appropriate fluorescein isothiocyanate fluorescence-labeled secondary antibody (1:200) (Sigma-Aldrich; Merck KGaA) for $40 \mathrm{~min}$ at $37^{\circ} \mathrm{C}$. 4',6-diamidino-2-phenylindole (DAPI) was used to stain the nucleus of astrocytes at $37^{\circ} \mathrm{C}$ for 4 min and cell climbing samples were observed under a fluorescent microscope (Nikon Corporation, Tokyo, Japan).

Evaluation of cell viability by MTT analysis. Cell viability was measured by MTT assay. A total of $24 \mathrm{~h}$ following reoxygenation, $20 \mu \mathrm{l}$ MTT solution was added ( $5 \mathrm{~g} / \mathrm{l})$ to the cell culture plate and after $4 \mathrm{~h}, 150 \mu \mathrm{l}$ DMSO was added then oscillated for $10 \mathrm{~min}$. Absorbance (A) value was measured by a microplate reader at a wavelength of $490 \mathrm{~nm}$. Finally, according to the following formula, the relative cell viability can be calculated: Relative cell viability $=$ experimental group/control group $\mathrm{x} 100 \%$.

Evaluation of cell cytotoxicity by LDH analysis. A total of $24 \mathrm{~h}$ following oxygen-glucose deprivation/reoxygenation, cell cytotoxicity was measured by LDH assay. Each group had $20 \mu \mathrm{l}$ cell culture fluid removed and LDH (U/l) was measured by colorimetry according to the protocol of the LDH assay kit. Finally, according to the following formula, the LDH leakage rate can be calculated: Relative cell cytotoxicity $=$ experimental group (U/l)/control group (U/l) x100\%. Through MTT experiment and $\mathrm{LDH}$ experiment the ideal concentration of Cur was identified and the optimum concentration was used in the following experiments.

Western blot analysis. Radioimmunoprecipiration assay lysis solution (Beyotime Institute of Biotechnology) was used to lyse the cells at $0^{\circ} \mathrm{C}$ for $20 \mathrm{~min}$, followed by centrifugation at $12,000 \times \mathrm{g}$ at $4^{\circ} \mathrm{C}$ for $10 \mathrm{~min}$ to collect protein supernatants. Using western blot analysis, the expression of MEK, p-MEK, ERK, p-ERK, CREB, p-CREB, Bcl-2 and Bax were measured. Samples $(30 \mu \mathrm{l})$ were separated by $12 \%$ SDS-PAGE then transferred to a nitrocellulose membrane. The membrane was blocked with $10 \%$ goat serum at $25^{\circ} \mathrm{C}$ for $2 \mathrm{~h}$ and the anti-rabbit primary antibodies against MEK (cat. no. SAB4502407), p-MEK (cat. no. HPA026430), ERK (cat. no. M7927), p-ERK (cat. no. M7927), CREB (cat. no. SAB4500444), 
p-CREB (cat. no. AV01026), Bcl-2 (cat. no. SAB4500003), and anti-mouse antibodies against Bax (cat. no. B8554) and $\beta$-actin (cat. no. A5441) $(1: 1,000)$ were added to the membrane overnight at $4{ }^{\circ} \mathrm{C}$. After washing, the membranes were incubated with anti-rabbit HRP-conjugated secondary antibodies (cat. no. AP182P; 1:2,000) at $37^{\circ} \mathrm{C}$ for $40 \mathrm{~min}$. Subsequently, the membranes were processed with an ECL kit to detect immune reactivity. Finally, images were analyzed with a Versa Doc Model 3000.

Statistical analysis. All data were expressed as the mean \pm standard deviation of the mean and were analyzed by SPSS version 24.0 for Windows (IBM Corps., Armonk, NY, USA). A one-way analysis of variance was used to compare the difference of measurement data among multiple groups. The post hoc test was performed using Tukey's post hoc method. The number of experimental repeats for each sample was 3 . Pairwise comparisons in multiple groups was conducted with the Student-Newman-Keuls method. $\mathrm{P}<0.05$ was considered to indicate a statistically significant difference.

\section{Results}

Cur can improve nerve damage symptoms in rats. A total of $24 \mathrm{~h}$ following ischemia/reperfusion, the neuroprotective effect of Cur was examined by evaluating neurological deficit scores and brain water content. As presented in Fig. 1, compared with the IR group, the IC group can significantly alleviate nerve damage symptoms $(\mathrm{P}<0.05)$, most notably in the IC group of $300 \mathrm{mg} / \mathrm{kg}$.

Cur can improve brain water content of IR rats. A total of $24 \mathrm{~h}$ following ischemia/reperfusion, the effect of Cur was examined by investigating brain water content. The results demonstrated that Cur can reduce cerebral edema. As presented in Fig. 2, IC groups can significantly reduce the brain water content $(\mathrm{P}<0.05)$, particularly the IC group at a dose of $300 \mathrm{mg} / \mathrm{kg}$. Through these two experiments, it was also demonstrated that neurological deficit scores and brain water content were decreased in a dose-dependent manner. In addition, only the IC group of $300 \mathrm{mg} / \mathrm{kg}$ were used in the rest of the experiments.

Cur can reduce the volume of a cerebral infarct in IR rats. A total of $24 \mathrm{~h}$ following ischemia/reperfusion, the infract volume was measured. As presented in Fig. 3, compared with the IR group, the IC group (IC, $300 \mathrm{mg} / \mathrm{kg}$ ) displayed significantly decreased pale-colored regions $(\mathrm{P}<0.01)$.

Cur can improve neuronal damage in CAl area by TEM. A total of $24 \mathrm{~h}$ following ischemia/reperfusion, changes in neurons were observed using TEM. As presented in Fig. 4, TEM of the SC group (magnification, x6,000) displayed normal neuronal structure and no significant alterations. However TEM of the IR group $(\mathrm{x} 6,000)$ revealed serious neuronal damage in the CA1 area, exhibiting nucleus electron density decreases, dissolution, cavitation, mitochondrial swelling and endoplasmic reticulum. Compared with the IR group, the IC group ( $300 \mathrm{mg} / \mathrm{kg}$; magnification, x6,000) exhibited less severe changes.

Cur can improve neuronal damage in the CAl region as demonstrated by $H \& E$ staining. A total of $24 \mathrm{~h}$ following

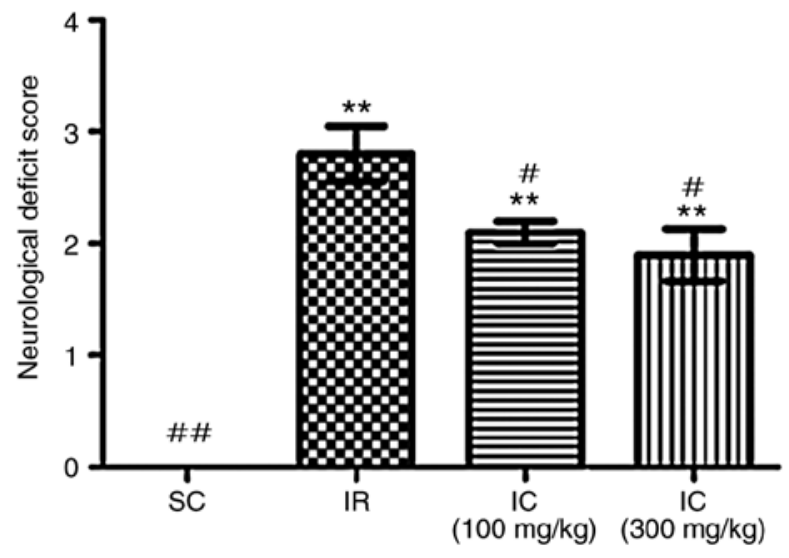

Figure 1. Effect of curcumin on the neurological deficit score following $24 \mathrm{~h}$ of transient middle cerebral artery occlusion $(\mathrm{n}=10)$. ${ }^{* *} \mathrm{P}<0.01 \mathrm{vs}$. $\mathrm{SC} ;{ }^{\#} \mathrm{P}<0.05$, ${ }^{\# \#} \mathrm{P}<0.01$ vs. IR. SC, sham group; IR, model; IC, curcumin group.

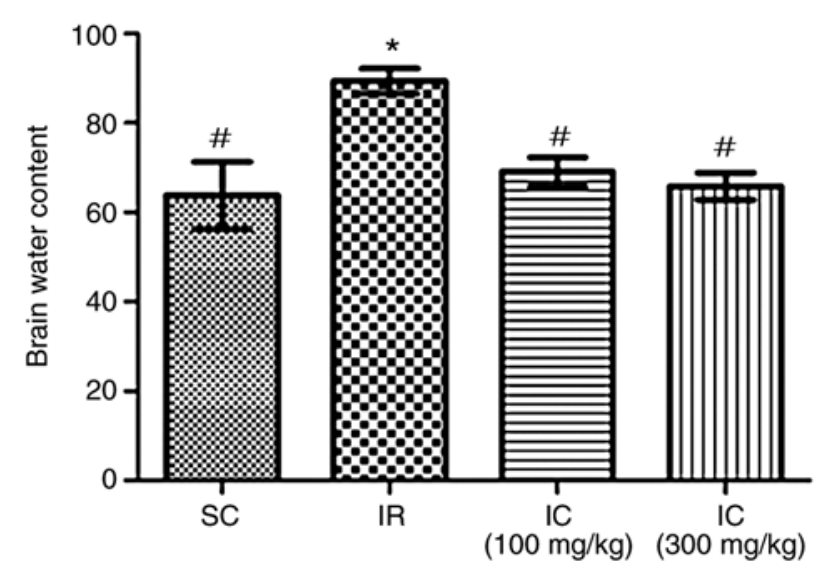

Figure 2. Effect of curcumin on the brain water content following $24 \mathrm{~h}$ of transient middle cerebral artery occlusion $(n=3)$. ${ }^{*} \mathrm{P}<0.05$ vs. $\mathrm{SC} ;{ }^{\#} \mathrm{P}<0.05$ vs. IR. SC, sham group; IR, model; IC, curcumin group.

ischemia/reperfusion, the IR group displayed abnormal cell structures and morphology. Specifically, cell body and nucleus condensation, stained nucleoli, and gaps around the cells were observed. As presented in Fig. 5, compared with the IR group, the IC group $(300 \mathrm{mg} / \mathrm{kg})$ significantly reduced abnormal cells $(\mathrm{P}<0.01)$.

Cur can reduce neuronal apoptosis in the hippocampal CAI region as demonstrated by TUNEL staining. A total of $24 \mathrm{~h}$ following ischemia/reperfusion, the TUNEL method was utilized to detect neuronal apoptosis of hippocampal CA1. In hippocampal CA1, normal cells were stained blue, but apoptotic cell nuclei were stained brown. As presented in Fig. 6, in the SC group, almost no TUNEL-positive cells were present in the hippocampal CA1 region. Compared with the SC group, the number of apoptotic cells in the IR group was significantly increased. However the IC group $(300 \mathrm{mg} / \mathrm{kg})$ displayed a significantly lower number of apoptotic cells $(\mathrm{P}<0.01)$.

Cur can increase the expression of p-MEK, MEK, $p$-ERK, $E R K, p-C R E B, C R E B, B c l-2$ and reduce the expression of Bax in vivo. The expression of p-MEK, MEK, p-ERK, ERK, p-CREB, CREB, Bcl-2 and Bax were detected by western 
A

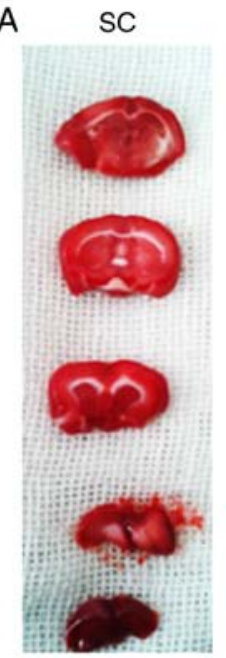

B

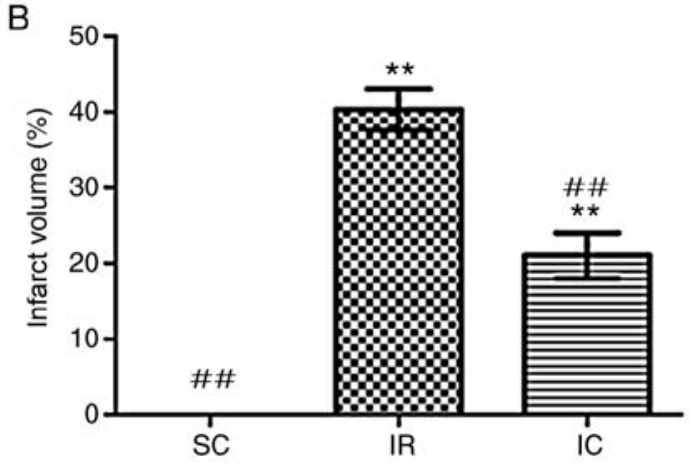

IC
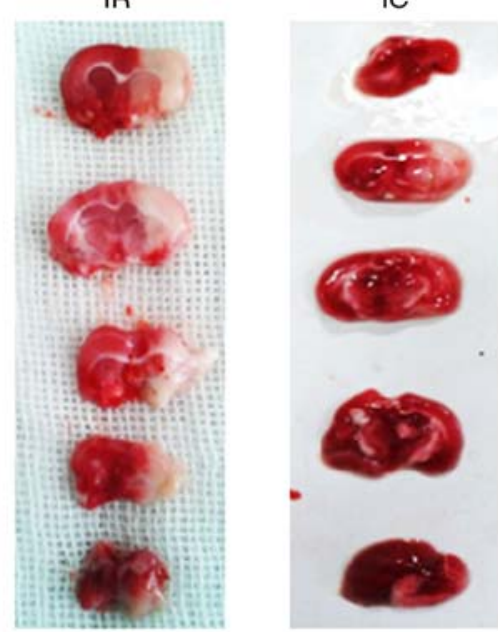

A

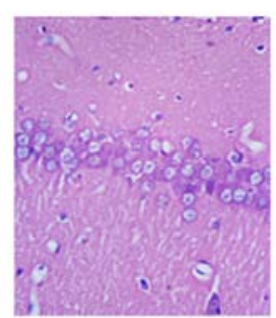

IR

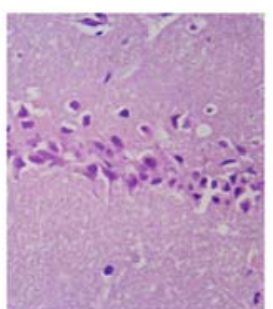

IC

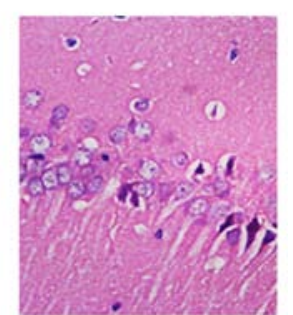

B

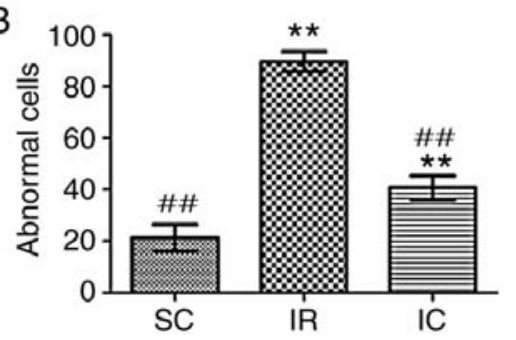

Figure 5. Effect of curcumin on the brain tissue morphology following $24 \mathrm{~h}$ of transient middle cerebral artery occlusion by hematoxylin and eosin staining $(\mathrm{n}=3$; magnification, $\mathrm{x} 400)$. (A) A representative image of the three independent experiments: SC; IR and IC ( $300 \mathrm{mg} / \mathrm{kg}$ ). (B) The number of abnormal cells in the three experiments was analyzed. ${ }^{* *} \mathrm{P}<0.01$ vs. the $\mathrm{SC}$; ${ }^{\# /} \mathrm{P}<0.01$ vs. IR. SC, sham group; IR, ischemia-reperfusion group; IC, curcumin treatment group.
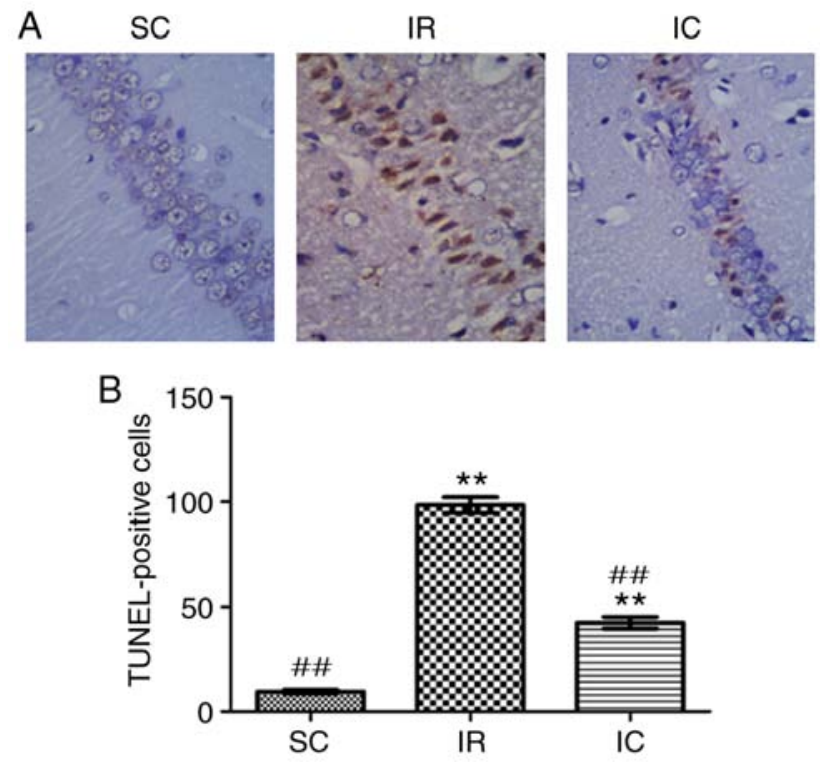

Figure 6. Effect of curcumin on the neurons apoptosis of hippocampal CA1 in rat $24 \mathrm{~h}$ of transient middle cerebral artery occlusion. (A) TUNEL staining of the neurons apoptosis in the rat brain $(n=3, x 400)$. (A) A representative image of the three independent experiments: SC; IR and IC $(300 \mathrm{mg} / \mathrm{kg})$. (B) The number of TUNEL-positive cells in the three experiments was analyzed. ${ }^{* *} \mathrm{P}<0.01$ vs. the $\mathrm{SC} ;{ }^{\# \#} \mathrm{P}<0.01$ vs. IR. SC, sham group; IR, ischemia-reperfusion group; IC, curcumin treatment group; TUNEL, terminal dexynucleotidyl transferase (TdT)-mediated dUTP.

notable green fluorescence was visible in the cell cytoplasm and projections, and nuclei appeared blue by DAPI staining. Furthermore, cytons were large and irregular with few cell projections of GFAP-positive cells. These characteristics were consistent with the morphology of astrocytes and distribution characteristics of GFAP, demonstrating that astrocytes were

present (Fig. 8).

Identification of astrocytes in vitro. Astrocytes were identi-
fied by immunofluorescence staining with astrocyte-specific
marker GFAP antibody. Under a fluorescent microscope, a

Identification of astrocytes in vitro. Astrocytes were identi-
fied by immunofluorescence staining with astrocyte-specific
marker GFAP antibody. Under a fluorescent microscope, a

Identification of astrocytes in vitro. Astrocytes were identi-
fied by immunofluorescence staining with astrocyte-specific
marker GFAP antibody. Under a fluorescent microscope, a

blotting $24 \mathrm{~h}$ following reperfusion in vivo. As presented in Fig. 7, it was demonstrated that the IC group $(300 \mathrm{mg} / \mathrm{kg}$ ) increased the expression of $\mathrm{p}-\mathrm{ERK}, \mathrm{p}-\mathrm{CREB}, \mathrm{Bcl}-2$ and reduced the levels of Bax significantly $(\mathrm{P}<0.01)$. 

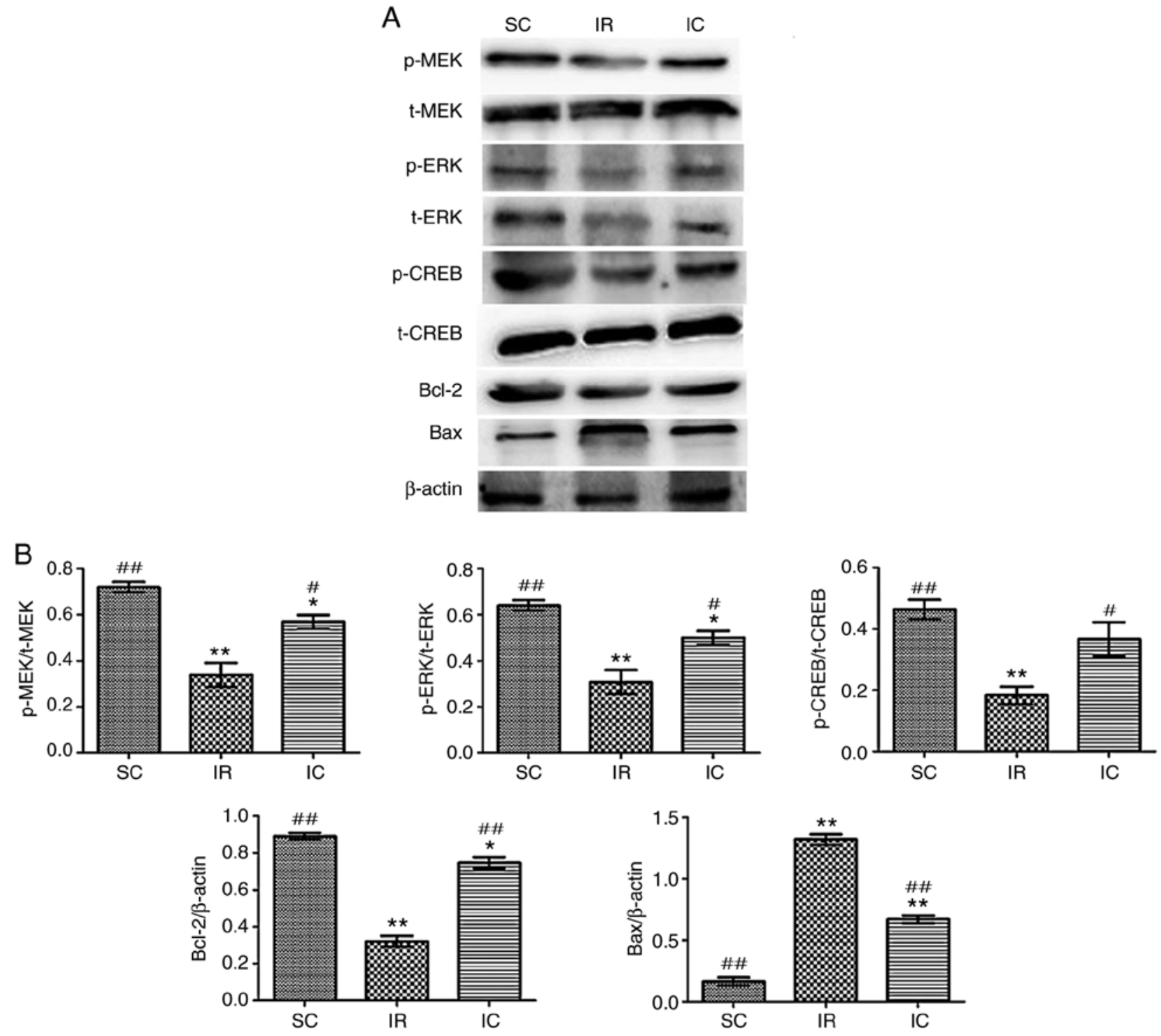

Figure 7. Effect of curcumin on the expression of p-MEK, MEK, p-ERK, ERK, p-CREB, CREB, Bcl-2 and Bax in vivo. (A) Western blot analysis of p-MEK, MEK, p-ERK, ERK, p-CREB, CREB, Bcl-2 and Bax (n=3). For p-MEK, p-ERK and p-CREB, t-MEK, t-ERK and t-CREB were used as references respectively. For Bcl-2 and Bax, $\beta$-actin was used as an internal reference. (B) The protein content in the three experiments was analyzed. ${ }^{*} \mathrm{P}<0.05$ and ${ }^{* *} \mathrm{P}<0.01$ vs. the SC; ${ }^{\#} \mathrm{P}<0.05$ and ${ }^{\# /} \mathrm{P}<0.01$ vs. IR. SC, sham group; IR, ischemia-reperfusion group; IC, curcumin treatment group; p, phosphorylated; Bcl, B-cell lymphoma; CREB, cAMP-response element binding protein; $t$, total; MEK, methyl ethyl ketone; ERK, extracellular signal regulated kinase; Bax, Bcl-2 associated X protein.

DAPI

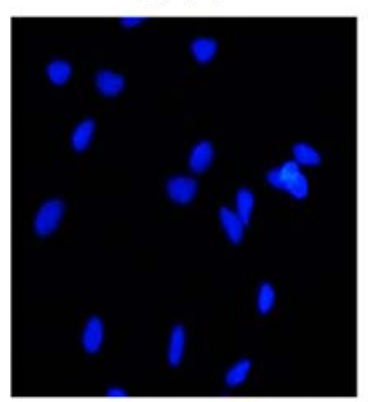

GFAP

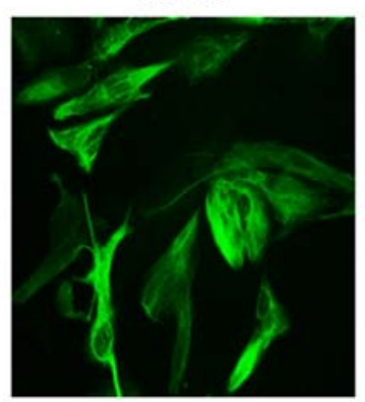

Overlay

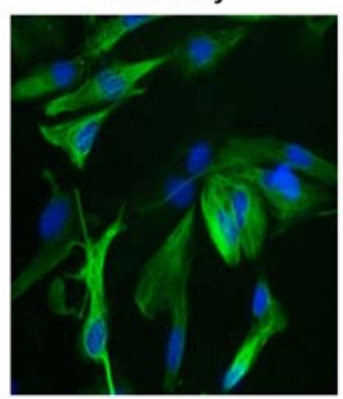

Figure 8. Immunofluorescence staining of GFAP (magnification, x200). These three images respectively represent: Nuclei staining by DAPI; GFAP staining; the figure was made by overlaying the previous two images. GFAP, glial fibrillary acidic protein; DAPI, 4',6-diamidino-2-phenylindole.

Cur can increase the viability of astrocytes in vitro. A total of $24 \mathrm{~h}$ following oxygen-glucose deprivation/reoxygenation, the effect of Cur on the viability of astrocytes was examined. As presented in Fig. 9. compared with the OGD/Reoxy group, the 


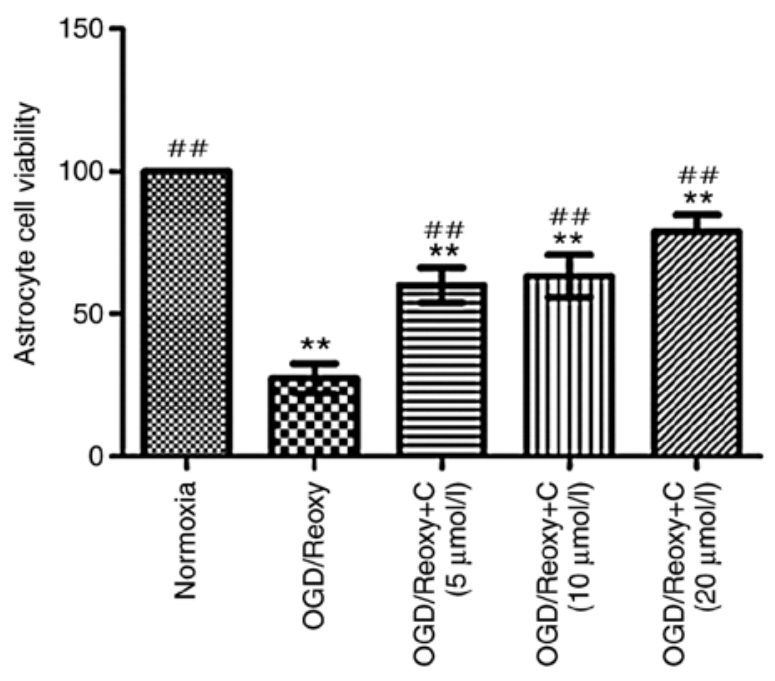

Figure 9. Effect of $\mathrm{C}$ on the viability of astrocytes following $24 \mathrm{~h}$ of OGD/Reoxy $(\mathrm{n}=6) .{ }^{* *} \mathrm{P}<0.01$ the normoxia group; ${ }^{\# \#} \mathrm{P}<0.01$ vs. the $\mathrm{OGD} /$ Reoxy group. OGD, oxygen glucose deprivation; Reoxy, reoxygenation; $\mathrm{C}$, curcumin.

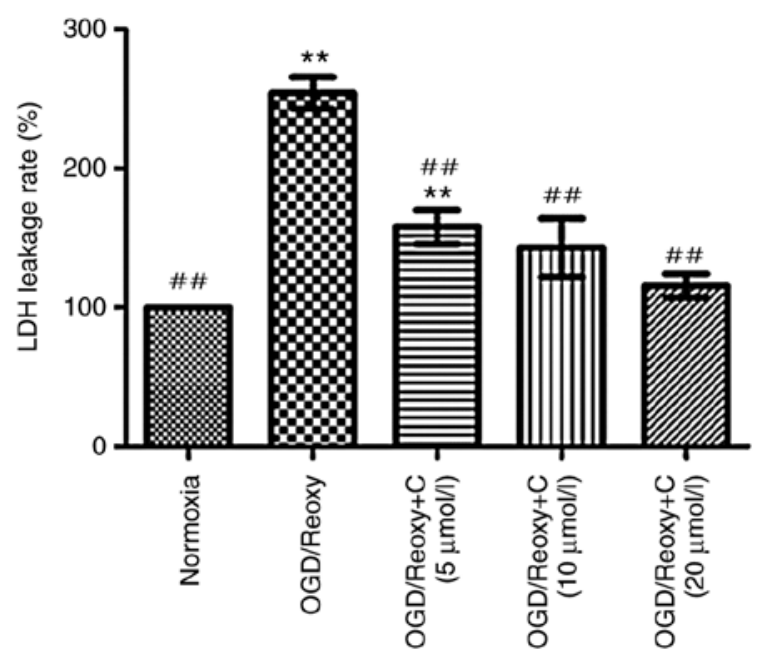

Figure 10. Effect of $\mathrm{C}$ on the LDH leakage rate (\%) following $24 \mathrm{~h}$ of OGD/Reoxy $(\mathrm{n}=6) .{ }^{* *} \mathrm{P}<0.01$ vs. the normoxia group; ${ }^{\# \#} \mathrm{P}<0.01$ vs. the OGD/ Rexory group. LDH, lactate dehydrogenase; OGD, oxygen glucose deprivation; Reoxy, reoxygenation; C, curcumin.

OGD/Reoxy + C groups significantly increased the astrocytes' viability $(\mathrm{P}<0.01)$, particularly the $\mathrm{OGD} /$ Reoxy $+\mathrm{C}$ group of $20 \mu \mathrm{mol} / 1$.

Cur can reduce LDH leakage rates. Following oxygen-glucose deprivation/reoxygenation for $24 \mathrm{~h}$, the effect of Cur on LDH leakage rates was examined. As presented in Fig. 10, compared with the OGD/Reoxy group, the OGD/Reoxy + C groups significantly reduced the $\mathrm{LDH}$ leakage rate $(\mathrm{P}<0.01)$, particularly the OGD/Reoxy $+\mathrm{C}$ group of $20 \mu \mathrm{mol} / \mathrm{l}$. Therefore, only C-treatment group of $20 \mu \mathrm{mol} / 1$ was used in other experiments.

Cur can increase the expression of $p-M E K, M E K, p-E R K$, ERK, P-CREB, CREB, Bcl-2 and reduce the expression of Bax in vitro. The expression of p-MEK, MEK, p-ERK, ERK, p-CREB, CREB, Bcl-2 and Bax were detected by western blotting for $24 \mathrm{~h}$ following reperfusion in vitro. As presented in Fig. 11, the IC groups $(20 \mu \mathrm{mol} / \mathrm{l})$ increased the expression of p-MEK, MEK, p-ERK, ERK, p-CREB, CREB, Bcl-2 and reduced the Bax levels significantly compared with the IR group $(\mathrm{P}<0.01)$. These results were consistent with the experimental results in vivo.

\section{Discussion}

Glial cells were first identified by Rudolf Virchow in 1846 (21). In the years since, a wide range of research has been conducted on the morphology and function of glial cells, especially astrocytes. Astrocytes were proved not to be simply inert cells, in contrast, they serve a very important role in the development of the nervous system, nerve tissue repair and regeneration, nerve and immune pathogenesis $(22,23)$.

There are also studies which indicate that activation of the MEK/ERK or ERK/CREB pathway may protect astrocytes from ischemic injury $(24,25)$. Cur is known to have neuroprotective properties in cerebral ischemia-reperfusion injury. However, the underlying molecular mechanisms are still poorly understood. The main focus of this study are the in vivo experiments that utilize the artery occlusion model in rats, however the hypothesis was further confirmed by conducting experimental studies in vitro. To the best of our knowledge this study is the first to investigate the association between Cur and the MEK/ERK/CREB signaling pathway in cerebral ischemia-reperfusion.

There is significant evidence demonstrating that activation of the MEK-ERK1/2 signaling pathway has a neuroprotective effect in cerebral ischemia-reperfusion injury (26). Additionally $\mathrm{Fu}$ et al (23) also demonstrated that increasing the expression of $\mathrm{p}-\mathrm{CREB}$ through MEK/ERK pathways can exert neuroprotective effects against ischemia. Previous studies have also confirmed that Bcl-2, BAD, CREB, glycogen synthesis kinase 3 and brain-derived neurotrophic factor are the MEK/ERK pathway downstream targets, and they serve a very important role in neuronal survival, development and maintaining the plasticity of neurons (27-29) ERK may also improve microcirculation and reduce neuronal apoptosis.

CREB and the active form p-CREB, are important nuclear transcription factors, which serve an indispensable role in the nervous system (30) and CREB activation is a key factor in neuroprotection against ischemic reperfusion damage. During a stroke, a variety of apoptosis regulatory gene products are activated (31). Among them, Bcl-2 is an important anti-apoptotic proteins and Bax is an important pro-apoptotic protein (32,33). Enhancing Bcl-2 and reducing Bax have been demonstrated to promote cell survival and promote a neuroprotective effect following focal cerebral ischemia $(34,35)$.

The present study suggests that Cur can improve neurological symptom scores and brain water content, (with the best effect at $300 \mathrm{mg} / \mathrm{kg}$ ) and enhance the activation of MEK, ERK and CREB. The expression of downstream signaling molecules Bcl-2 is regulated by CREB. As indicated above, the expression of $\mathrm{Bcl}-2$ was enhanced and Bax was reduced. The results of the TUNEL assay also verified the neuroprotective effects in terms of pathomorphology. In hippocampal CA1 of the rats, the results of TUNEL demonstrated that in the SC group, almost no TUNEL-positive cells were identified in the hippocampal CA1 region. However in the IR group, TUNEL-positive cells were increased. Following treatment 


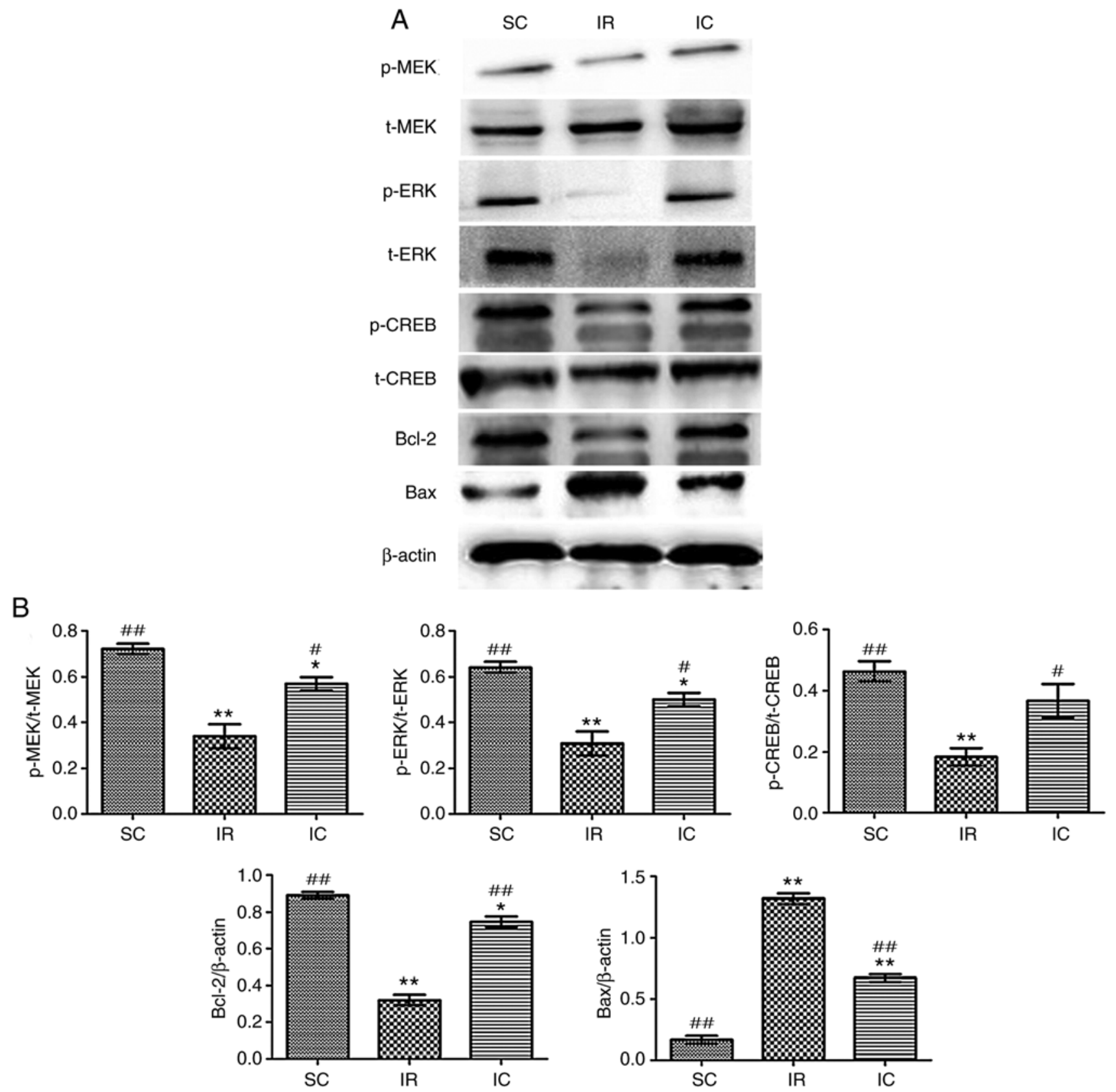

Figure 11. Effect of curcumin on the expression of p-MEK, MEK, p-ERK, ERK, p-CREB, CREB, Bcl-2 and Bax in vitro. (A) Western blot analysis of p-MEK, MEK, p-ERK, ERK, p-CREB, CREB, Bcl-2 and Bax (n=3). For p-MEK, p-ERK and p-CREB, t-MEK, t-ERK and t-CREB were used reference respectively. For Bcl-2 and Bax, $\beta$-actin was used as an internal reference. (B) The protein contents in the three experiments were analyzed. ${ }^{*} \mathrm{P}<0.05$ and ${ }^{* * *} \mathrm{P}<0.01 \mathrm{vs}$. SC; ${ }^{\#} \mathrm{P}<0.05$ and ${ }^{\# \#} \mathrm{P}<0.01$ vs. IR. SC, sham group; IR, ischemia-reperfusion group; IC, curcumin treatment group; p, phosphorylated; Bcl, B-cell lymphoma; CREB, cAMP-response element binding protein; t, total; MEK, methyl ethyl ketone; ERK, extracellular signal regulated kinase; Bax, Bcl-2 associated X protein.

with Cur (300 mg/kg), TUNEL-positive cells were reduced. The results of neurobehavioral scores, TEM and H\&E, were also consistent with these findings.

In conclusion, Cur can protect rats from focal cerebral ischemia-reperfusion injury and this effect may be carried out through the MEK/ERK/CREB pathway.

\section{Acknowledgements}

The authors would like to thank Professor Zhi Dong for guidance and the Chongqing Medical University Laboratory of Biochemistry and Molecular Pharmacology for providing the platform for the experiment.

\section{Funding}

The present study was supported financially by the Chongqing Science and Technology Commission (grant nos. CSTC2016jcyjA0268, CSTC2018jcyjAX0821 and CSTC2018jxj1130009) and the Chongqing Medical and Pharmaceutical College Scientific Research Projects (grant no. ygz2018302).

\section{Availability of data and materials}

All data generated or analyzed during this study are included in this published article. 


\section{Authors' contributions}

YH and JL conceived and designed the study. LX, LD and YS performed the experiments. RS analyzed the data. LX wrote and revised the paper. $\mathrm{YH}$ and $\mathrm{JL}$ reviewed and edited the manuscript. All authors read and approved the manuscript.

\section{Ethics approval and consent to participate}

Experimental protocols were all approved by the Chongqing Medical and Pharmaceutical College's Institutional Animal Care and Use Committee.

\section{Patient consent for publication}

Not applicable.

\section{Competing interests}

The authors declare that they have no competing interests.

\section{References}

1. Durai Pandian J, Padma V, Vijaya P, Sylaja PN and Murthy JM: Stroke and thrombolysis in developing countries. Int J Stroke 2 17-26, 2007.

2. Moskowitz MA, Lo EH and Iadecola C: The science of stroke: Mechanisms in search of treatments. Neuron 67: 181-198, 2010.

3. Benjamin EJ, Virani SS, Callaway CW, Chamberlain AM, Chang AR, Cheng S, Chiuve SE, Cushman M, Delling FN, Deo R, et al: Heart disease and stroke statistics-2018 update: A report from the American heart association statistics committee and stroke statistics subcommittee. Circulation 137: e67-e492, 2018.

4. Fogelholm R: Editorial comment-Explanations for international trends in stroke mortality. Stroke 34: 1833-1840, 2003.

5. Nagahiro S, Uno M, Sato K, Goto S, Morioka M and Ushio Y: Pathophysiology and treatment of cerebral ischemia. J Med Invest 45: 57-70, 1998.

6. Bates B, Choi JY, Duncan PW, Glasberg JJ, Graham GD, Katz RC, Lamberty K, Reker D, Zorowitz R, US Department of Defense and Department of Veterans Affairs: Veterans affairs/department of defense clinical practice guideline for the management of adult stroke rehabilitation care: Executive summary. Stroke 36: 2049-2056, 2005.

7. Grotta $\mathbf{J}$ and Marler J: Intravenous rt-PA: A tenth anniversary reflection. Surg Neurol 68: S12-S16, 2007.

8. Pan J, Konstas AA, Bateman B, Ortolano GA and Pile-Spellman J: Reperfusion injury following cerebral ischemia: Pathophysiology, MR imaging, and potential therapies. Neuroradiology 49: 93-102, 2007.

9. Minnerup J, SutherlandBA, Buchan AM and Kleinschnitz C: Neuroprotection for stroke: Current status and future perspectives. Int J Mol Sci 13: 11753-11772, 2012.

10. Love S: Apoptosis and brain ischaemia. Prog Neuropsychopharmacol Biol Psychiatry 27: 267-282, 2003.

11. Sugawara T, Fujimura M, Noshita N, Kim W, Saito A Hayashi T, Narasimhan P, Maier CM and Chan Pak H: Neuronal death/survival signaling pathways in cerebral ischemia. NeuroRx 1: 17-25, 2004.

12. Zhao Y, Gui W, Niu F and Chong S: The MAPK signaling pathways as a novel way in regulation and treatment of parasitic diseases. Disease 7: E9, 2019.

13. Selcher JC, Atkins CM, Trzaskos JM, Paylor R and Sweatt JD: A necessity for MAP kinase activation in mammalian spatial learning. Learn Mem 6: 478-490, 1999.

14. Cheng CY, Lin JG, Su SY, Tang NY, Kao ST and Hsieh CL: Electroacupuncture-like stimulation at baihui and dazhui acupoints exerts neuroprotective effects through activation of the brain-derived neurotrophic factor-mediated MEK1/2/ERK/1/2/p90RSK/bad signaling pathway in mild transient focal cerebral ischemia in rats. BMC Complement Altern Med 14: 92, 2014.
15. Li J, Li X, Bi H and Li B: The MEK/ERK/CREB signaling pathway is involved in atrazine induced hippocampal neurotoxicity in sprague dawley rats. Ecotoxicol Environ Saf 170: 673-681, 2019.

16. Zuo H, Lin T, Wang W, Peng R, Wang S, Gao Y, Xu X, Zhao L, Wang $S$ and $S u$ Z: RKIP regulates neural cell apoptosis induced by exposure to microwave radiation partly through the MEK/ERK/CREB pathway. Mol Neurobiol 51: 1520-1529, 2014.

17. Zhao X, Xu Y, Zhao Q, Chen CR, Liu AM and Huang ZL: Curcumin exerts antinociceptive effects in a mouse model of neuropathic pain: Descending monoamine system and opioid receptors are differentially involved. Neuropharmacology 62: 843-854, 2012.

18. Kim KT, Kim MJ, Cho DC, Park SH, Hwang JH, Sung JK, Cho HJ and Jeon Y: The neuroprotective effect of treatment with curcumin in acute spinal cord injury: Laboratory investigation. Neurol Med Chir 54: 387-394, 2014.

19. Gazal M, Valente MR, Acosta BA, Kaufmann FN, Braganhol E, Lencina CL, Stefanello FM, Ghisleni G and Kaster MP: Neuroprotective and antioxidant effects of curcumin in a ketamine-induced model of mania in rats. Eur J Pharmacol 724: 132-139, 2014.

20. Longa EZ, Weinstein PR, Carlson S and Cummins R: Reversible middle cerebral artery occlusion without craniectomy in rats. Stroke 20: 84-91, 1989.

21. Nilsson M, Thorlin T, Blomstrand F and Hansson E: The starshaped cells. Astrocytes are involved in the pathogenesis and progress of neurological diseases. Lakartidningen 97: 3604-3610, 2000.

22. Ridet JL, Malhotra SK, Privat A and Gage FH: Reactive astrocytes: Cellular and molecular cues to biological function. Trends Neurosci 20: 570-577, 1997.

23. Fu J, Xue R, Gu J, Xiao Y, Zhong H, Pan X and Ran R: Neuroprotective effect of calcitriol on ischemic/reperfusion injury through the NR3A/CREB pathways in the rat hippocampus. Mol Med Rep 8: 1708-1714, 2013.

24. Jiang Z, Zhang Y, Chen X, Lam PY, Yang H, Xu Q and Yu AC: Activation of Erk1/2 and Akt in astrocytes under ischemia. Biochem Biophys Res Commun 294: 726-733, 2002.

25. Zhou B, Chen D, Xu H and Zhang X: Proliferation of rabbit chondrocyte and inhibitioof IL-1 $\beta$-induced apoptosis through MEK/ERKsignaling by statins. In Vitro Cell Dev Biol Anim 53: 124-131, 2017.

26. YuZ,Cai M,LiX,ZhangJ,WuT,Yang F,ZhuW,Xiang Y,Zhang W, Xiang J and Cai D: Neuroprotective effects of Tongxinluo on focal cerebral ischemia and reperfusion injury inrats associated with the activation of the MEK1/2/ERK1/2/p90RSK signaling pathway. Brain Res 1685: 9-18, 2018.

27. Huang EJ and Reichardt LF: Neurotrophins: Roles in neuronal development and function. Annu Rev Neurosci 24: 677-736, 2001.

28. Marinissen MJ and Gutkind JS: G-protein-coupled receptors and signaling. Networks: Emerging paradigms. Trends Pharmacol Sci 22: 368-376, 2001

29. Weeber EJ and Sweatt JD: Molecular neurobiology of human cognition. Neuron 33: 845-848, 2002.

30. Sakamoto K, Karelina K and Obrietan K: CREB: A multifaceted regulator of neuronal plasticity and protection. J Neurochem 116: $1-9,2011$.

31. Lipton P: Ischemic cell death in brain neurons. Physiol Rev 79: 1431-1568, 1999.

32. Brambrink AM, Schneider A, Noga H, Astheimer A, Gotz B, Korner I, Heimann A, Welschof M and Kempski O: Tolerance-Inducing dose of 3-nitropropionic acid modulates bcl- 2 and bax balance in the rat brain: A potential mechanism of chemical preconditioning. J Cereb Blood Flow Metab 20: 1425-1436, 2000.

33. Ajami M, Eghtesadi S, Razaz JM, Kalantari N, Habibey R, Nilforoushzadeh MA, Zarrindast $M$ and Pazoki-Toroudi $H$ : Expression of $\mathrm{Bcl}-2$ and $\mathrm{Bax}$ after hippocampal ischemia in DHA +EPA treated rats. Neurol Sci 32: 811-818, 2011.

34. Zhu Y, Bu Q, Liu X, Hu W and Wang Y: Neuroprotective effect of TAT-14-3-3epsilon fusion protein against cerebral ischemia/reperfusion injury in rats. PLoS One 9: e93334, 2014.

35. Boucher MJ, Duchesne C, Laine J, Morisset J and Rivard N: cAMP protection of pancreatic cancer cells against apoptosis induced by ERK inhibition. Biochem Biophys Res Commun 285: 207-216, 2001. 